\title{
Exclusive production of one and two heavy quarkonia in nuclear collisions
}

\section{Wolfgang Schäfer ${ }^{* \dagger}$}

Institute of Nuclear Physics PAN, Kraków, Poland

E-mail: Wolfgang.Schafer@ifj.edu.pl

\section{Anna Cisek}

Institute of Physics, University of Rzeszów, Rzeszów, Poland

E-mail: acisek@univ.rzeszow.pl

\section{Antoni Szczurek}

Institute of Physics, University of Rzeszów, Rzeszów, Poland

Institute of Nuclear Physics PAN, Kraków, Poland

E-mail: Antoni.Szczurek@ifj.edu.pl

\begin{abstract}
High energy protons or ions are the source of a flux of Weizsäcker-Williams photons, which can be utilized to study the photoproduction of vector mesons also at the Tevatron and LHC colliders. We discuss how information on the small- $x$ gluon distribution in protons and nuclei can be obtained. We present our calculations based on a $k_{\perp}$-factorization approach which allows us to construct the unintegrated glue of a nucleus from the free-nucleon one.

We also discuss the production of $J / \psi$ pairs in nucleus-nucleus collisions via photon-photon fusion. Here we show that at presently accessible energies, the $J / \psi$-pair production is dominated by the "box-diagram" mechanism, i.e. the exchange of $c \bar{c}$ pairs in the crossed channels.
\end{abstract}

XXI International Workshop on Deep-Inelastic Scattering and Related Subject-DIS2013,

Marseilles, France

\footnotetext{
* Speaker.

$\dagger$ This work was partially supported by the Polish Ministry of Science and Higher Education (MNiSW) grant No. DEC-2011/01/B/ST2/04535.
} 


\section{Introduction}

Diffractive dissociation of (virtual) photons arises from the quasielastic scattering of the photon's color-dipole Fock states [1]. Exclusive diffractive vector meson production on the hadron target $\mathrm{h}(h=p, A), \gamma^{*}\left(Q^{2}\right) h \rightarrow V h$, is a special case, where the final state $q \bar{q}$-pair ends up in the bound state. A salient property of this process is that the transtion $\gamma \rightarrow V$, is dominated by dipole sizes $r_{S} \sim 1 / \sqrt{Q^{2}+M_{V}^{2}}$, where $Q^{2}$ is the photon's virtuality, and $M_{V}$ the mass of $V$ [2]. In particular this implies that the mass of a heavy vector meson can serve as a hard scale even in situations where momentum transfer $t$ and/or photon virtuality $Q^{2}$ are small. If the scanning radius $r_{S}$ is sufficiently small, the diffractive amplitude becomes proportional to the integrated glue of the target:

$$
A\left(\gamma^{*} h \rightarrow V h\right) \propto r_{S}^{2} \sigma\left(x, r_{S}\right) \propto\left(Q^{2}+M_{V}^{2}\right)^{-2} x g\left(x, Q^{2}+M_{V}^{2}\right) .
$$

The dependence of the diffractive cross section on the $\gamma^{*} h$-cms energy $W$ is then driven by the dependence of the proton's gluon distribution on $x=\frac{Q^{2}+M_{V}^{2}}{W^{2}}$ at the relevant hard scale. The effective Pomeron-intercept rising with $Q^{2}+M^{2}$ is indeed born out by HERA data, see e.g. the review [3]. The important point for us in the applications presented below is the sensitivity to the gluon dynamics in the target. In the practical calculations we do not rely on the oversimplified form (1) of the diffractive amplitude. Instead we use a $k_{\perp}$-factorization framework [3] which fully accounts for transverse momenta of gluons as well as for quantum motion of quarks in the bound states. The former is quantified by the unintegrated gluon distribution of the target, while the latter is accounted for by the light-cone wave-function of the bound state.

\section{Exclusive vector mesons in $p p$ and $A A$ collisions}

Exclusive central production mechanisms lead to very clean events, where protons/nuclei disappear intact into the beamlines (ideally they would be tagged by forward detectors), and the produced system is completely measured in the central detectors. The theoretical description of such processes has to be based on the fusion of various $t$-channel exchanges, with modifications due to absorptive/rescattering corrections. For a vector meson in the final state, one of the exchanges must be $C$-odd. The first obvious contribution is the photon-Pomeron fusion, which is calculable precisely in terms of the photoproduction amplitude on the hadron target. Example results of such calculations for proton-proton scattering are shown in Fig.1. Two different unintegrated gluon distributions were used, which both reasonably well describe inclusive $F_{2}$ data from HERA. Lines labelled 'IN' use an unintegrated gluon distribution [4] which does not contain any explicit effects of nonlinear evolution/gluon fusion. The label 'KS' designates an unintegrated glue [5] which was obtained by solving a nonlinear evolution equation of the Balitsky-Kovchegov type. Dashed lines are obtained neglecting absorptive corrections, while the solid lines include them. Before we turn to nucleus-nucleus collisions, we need to briefly explain how to obtain the diffractive photoproduction amplitudes on the nucleus: what replaces the Pomeron exchange? At not too small $x \sim x_{A}=\left(R_{A} m_{p}\right)^{-1} \sim 0.01$ the $\bar{q} q$ Fock state of the photon interacts coherently with the whole nucleus. The corresponding dipole scattering amplitude can be constructed from Glauber-Gribov theory:

$$
\Gamma\left(b, x_{A}, r\right)=1-\exp \left[-\sigma\left(x_{A}, r\right) T_{A}(b) / 2\right]=\int d^{2} \kappa\left[1-e^{i \kappa r}\right] \phi\left(b, x_{A}, \kappa\right) .
$$



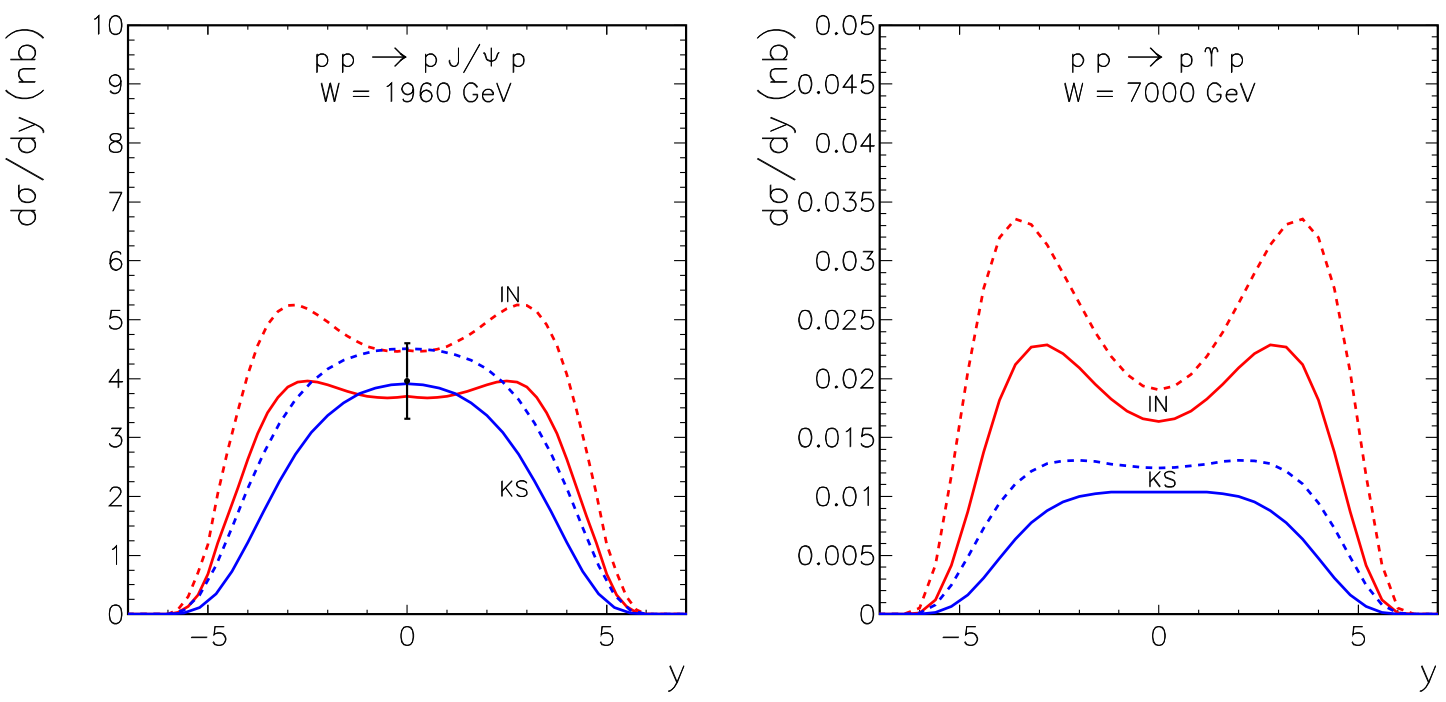

Figure 1: Exclusive production of $J / \psi$-mesons in $p \bar{p}$ collisions at the Tevatron (left panel) and of $\Upsilon$ in $p p$-collisions at LHC energy. The data point is from CDF [6]. Dashed lines do not include absorptive corrections, while solid lines do. Calculations from [7].

Following [8] it can be related to a nuclear coherent glue per unit area in impact parameter space:

$$
\phi\left(b, x_{A}, \kappa\right)=\sum w_{j}\left(b, x_{A}\right) f^{(j)}\left(x_{A}, \kappa\right), f^{(1)}(x, \kappa)=\frac{4 \pi \alpha_{S}}{N_{c}} \frac{1}{\kappa^{4}} \frac{\partial G\left(x, \kappa^{2}\right)}{\partial \log \left(\kappa^{2}\right)} .
$$

Here $\left.f^{(j}\right)(\kappa)$ is the $j$-fold convolution of the nuclear glue, the weight factors $w_{j}$ can be found in [9]. The so-constructed nuclear glue contains the effects of multiple scatterings of the $q \bar{q}$ state. At still smaller $x$ one must include the effects of (nonlinear) QCD-evolution. This is done by explicitly including the $q \bar{q} g$-state and its intranuclear rescatterings. In momentum space there emerges the triple-Pomeron vertex in the form of [10], and with some reservations these corrections can be identified with the gluon shadowing. A similar procedure has been previously successfully used for nuclear structure functions and continuum diffraction in [11]. A useful quantity is the ratio of the total diffractive cross section to its value in impulse approximation:

$$
R_{\mathrm{coh}}(W)=\frac{\sigma(\gamma A \rightarrow V A ; W)}{\sigma_{I A}(\gamma A \rightarrow V A ; W)}, \sigma_{I A}=4 \pi \int d^{2} b T_{A}^{2}(b) \frac{d \sigma(\gamma N \rightarrow V N)}{d t} \mid t=0 .
$$

It is this ratio which is calculated within the model, the $\gamma A$-cross section is then evaluated using a parametrization of data for $\frac{d \sigma(\gamma N \rightarrow V N)}{d t} \mid t=0$. Notice that in the crude approximation of eq.(1), the ratio $R_{\text {coh }}=\left(g_{A}\left(x, \bar{Q}^{2}\right) /\left(A \cdot g_{N}\left(x, \bar{Q}^{2}\right)\right)\right)^{2}$ is the square of the nuclear gluon shadowing ratio. We show our calculations of $R_{\text {coh }}$ for $\Upsilon$ and $J / \psi$ mesons as well as for $d \sigma(A A \rightarrow A A J / \psi) / d y$ in Fig.2. [12].

\section{Exclusive production of $J / \psi$-pairs via $\gamma-\gamma$ fusion}

This section is based on work done in collaboration with Sergey Baranov and Mariola KłusekGawenda [13]. The recent paper by the ALICE collaboration [12] contains an intriguing statement 

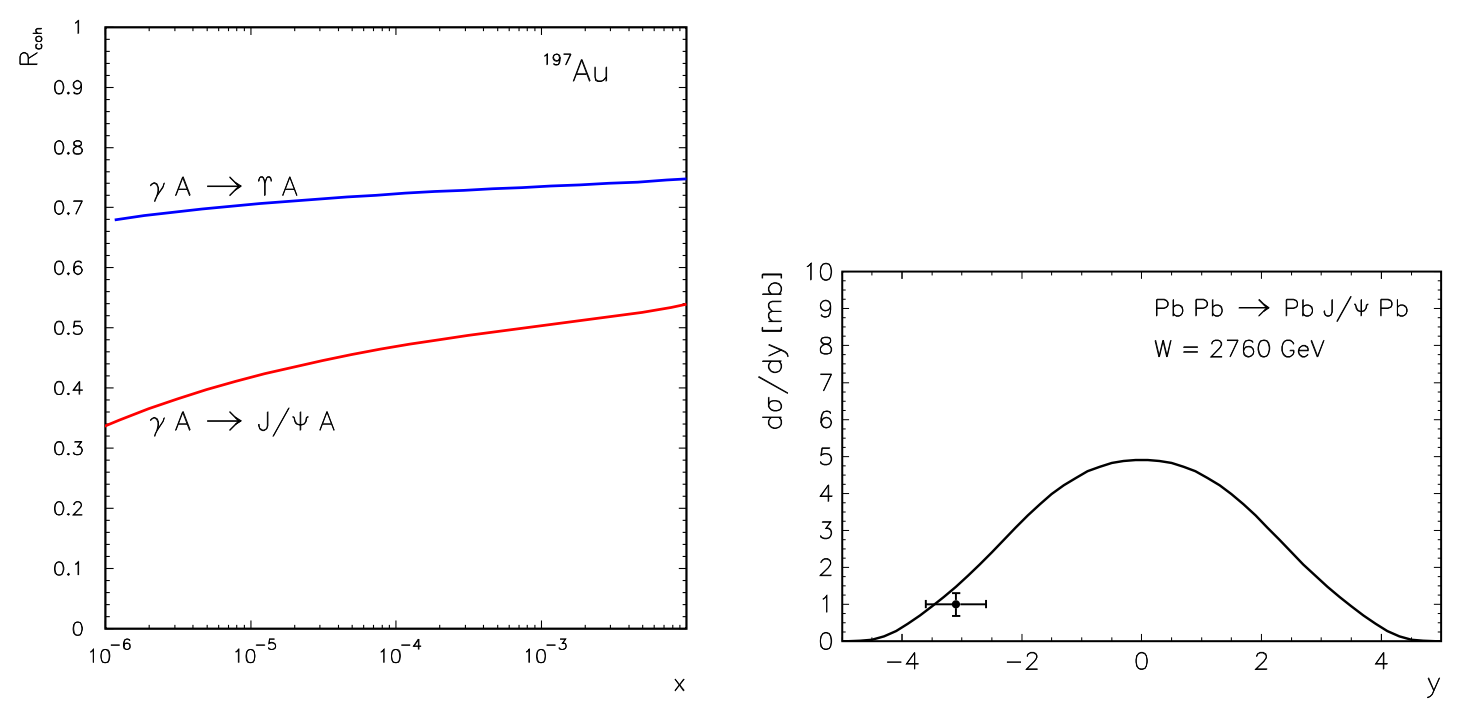

Figure 2: Left panel: $R_{\text {coh }}$ for $\Upsilon$ and $J / \psi$. Right panel: $d \sigma / d y$ for exclusive coherent production of $J / \psi$ in $\mathrm{Pb}-\mathrm{Pb}$ collisions at LHC energy [9] . The data point is from the ALICE collaboration [12]
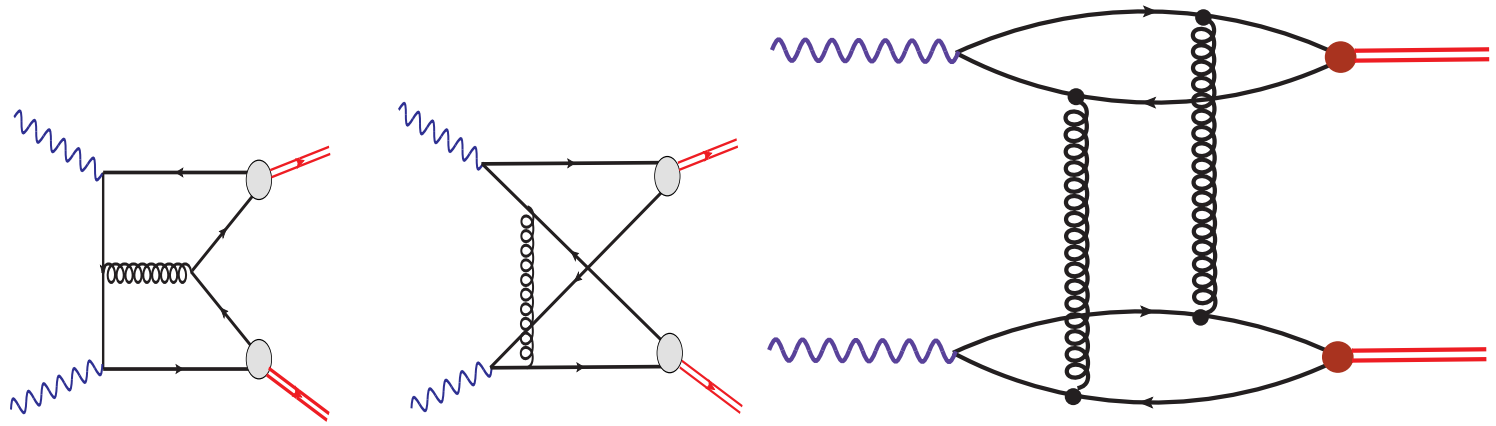

Figure 3: Left: Box diagram mechanism for $J / \psi$-pair production. Right: the two-gluon exchange contribution which dominates at high $\gamma-\gamma \mathrm{cm}$-energies.

on the presence of "coherent events" with two $J / \psi$-mesons in the final state. Two $J / \psi$ 's can be produced in $\gamma-\gamma$-fusion. This production mechanism contributes to the same event topology as coherent diffractive mechanism, only the total transverse momentum of the produced system will be substantially smaller. At high $\gamma-\gamma$ center of mass energies the production of vector meson pairs is the gold-plated probe of the dipole-dipole cross section which is of fundamental interest for the QCD-Pomeron physics. In practice, at accessible energies in nucleus-nucleus collisions however $J / \psi$-pair production is dominated by the "box"-type quark exchange diagrams (see Fig.3). In Fig. 4 we show the results from [13]. The two-gluon exchange amplitude strongly depends on the treatment of intrinsic motion in the bound state and has a surprisingly strong infrared sensitivity. The right panel shows that the photon-fusion mechanism of $J / \psi$-pair production is many orders smaller than coherent single- $J / \psi$ production. Pair production of $J / \psi$ 's may therefore be dominated 

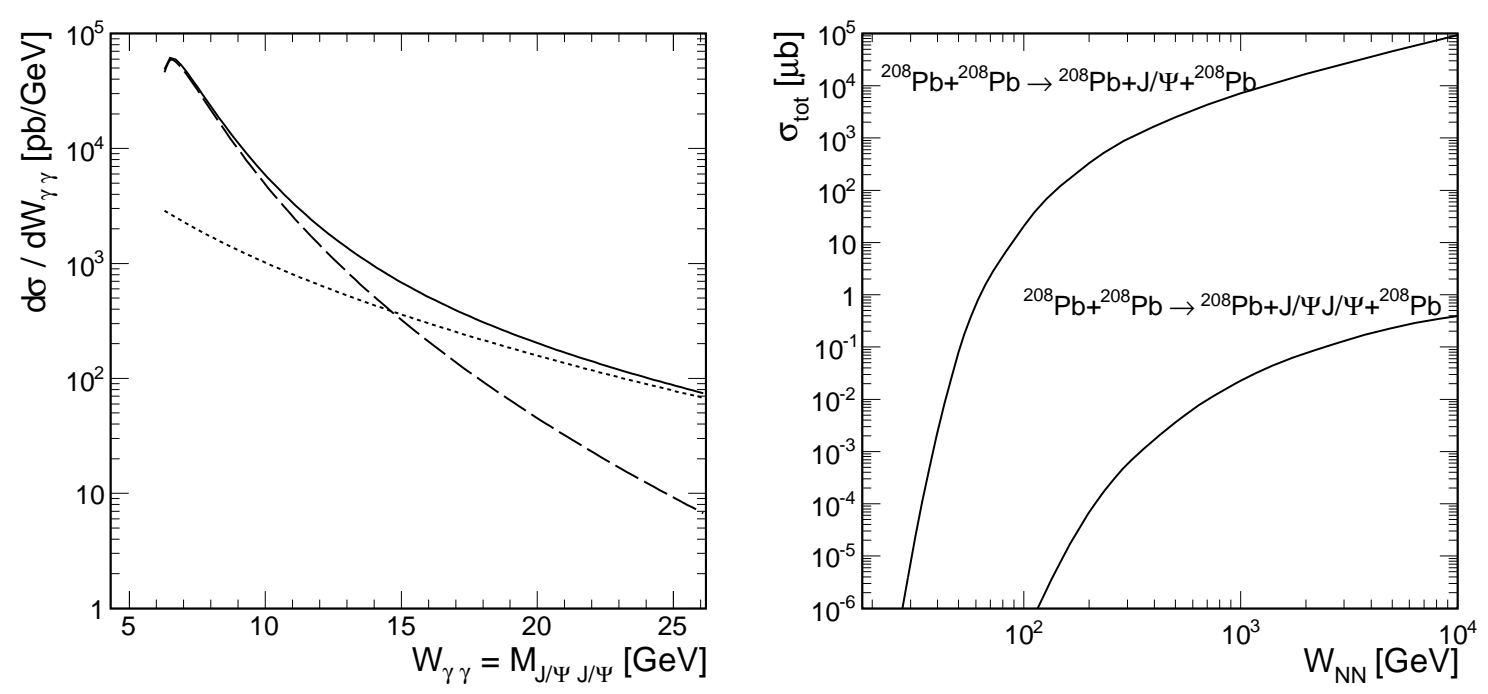

Figure 4: Left: Cross section for $A A \rightarrow A A J / \psi J / \psi$ differential in the invariant mass of the $J / \psi J / \psi$ pair at LHC energy $\sqrt{s_{N N}}=2.76 \mathrm{TeV}$. The long-dashed curve shows the contribution of the box-diagram mechanism, while the dotted curve is the two-gluon exchange contribution. Right: total cross sections.

by multiple coherent production.

\section{References}

[1] N. Nikolaev and B. G. Zakharov, Z. Phys. C 53 (1992) 331.

[2] N. N. Nikolaev, Comments Nucl. Part. Phys. 21 (1992) 41; B. Z. Kopeliovich, J. Nemchick, N. N. Nikolaev and B. G. Zakharov, Phys. Lett. B 309 (1993) 179; J. Nemchik, N. N. Nikolaev, E. Predazzi and B. G. Zakharov, Z. Phys. C 75 (1997) 71 .

[3] I. P. Ivanov, N. N. Nikolaev and A. A. Savin, Phys. Part. Nucl. 37 (2006) 1.

[4] I. P. Ivanov and N. N. Nikolaev, Phys. Rev. D 65 (2002) 054004 [hep-ph/0004206].

[5] K. Kutak and A. M. Staśto, Eur. Phys. J. C 41 (2005) 343.

[6] T. Aaltonen et al. [CDF Collaboration], Phys. Rev. Lett. 102 (2009) 242001.

[7] A. Cisek, Exclusive processes with large rapidity gaps in the formalism of unintegrated gluon distributions, (PhD thesis, The Henryk Niewodniczański Institute of Nuclear Physics, Polish Academy of Sciences, Kraków, 2012)

[8] N. N. Nikolaev, W. Schäfer and G. Schwiete, Phys. Rev. D 63 (2001) 014020.

[9] A. Cisek, W. Schäfer and A. Szczurek, Phys. Rev. C 86 (2012) 014905.

[10] N. N. Nikolaev and W. Schäfer, Phys. Rev. D 74 (2006) 014023 [hep-ph/0604117].

[11] N. N. Nikolaev, W. Schäfer, B. G. Zakharov and V. R. Zoller, JETP Lett. 84 (2007) 537.

[12] B. Abelev et al. [ALICE Collaboration], Phys. Lett. B 718 (2013) 1273 [arXiv:1209.3715 [nucl-ex]].

[13] S. Baranov, A. Cisek, M. Kłusek-Gawenda, W. Schäfer and A. Szczurek, Eur. Phys. J. C 73 (2013) 2335 [arXiv:1208.5917 [hep-ph]]. 